\title{
THE SHEARING BEHAVIOUR OF A SUGAR AGGREGATE
}

\author{
Catherine A. Davy ${ }^{1}$, M. D. Bolton ${ }^{2}$, N. A. Fleck ${ }^{2}$
}

October 2003

1: GeM, Civil Engineering and Mechanics Research Institute

1 rue de la Noë,

44321 Nantes Cedex 3, France

2: Cambridge University Engineering Department, Trumpington Street,

Cambridge CB2 1PZ, UK 


\section{Abstract}

To better understand the behaviour of sugar within industrial centrifuges or silos, drained triaxial tests were performed on loose, dry or moist sugar aggregates under low confining pressures $(15$ to $90 \mathrm{kPa}$ ). Particular attention is paid to test reproducibility and grain damage level. Rowe's stress-dilatancy theory is used to improve characterisation of the critical state, at which the aggregate of grains could participate in frictional flow at constant volume. The effects of strain rate, mean grain size and moisture upon internal friction and dilatancy are each investigated. Additionally, initial elastic properties are identified.

Keywords Granular materials, compression test, internal friction, slip, yield phenomena. 


\section{Introduction}

Refined white sugar is obtained by the centrifugal separation of sugar crystals from molasses in several steps. Although continuous centrifuges have recently been improved [1], the final and most arduous centrifugal stage remains too demanding for them, and batch machines remain the only viable option. The development of a High Grade Continuous Centrifuge would represent a significant processing development for the Sugar Industry, but this rests upon developments in the fundamental understanding and modelling of the compaction and flow of sugar within a centrifuge. It is to be noted that although the sugar mix can be assimilated to a slurry at the entrance of the centrifuge, the centrifugal process turns it rapidly into a moist granular material as it flows along the centrifuge basket. Another characteristic of that type of aggregate is its looseness, which is also encountered in other industrial conditions, such as heap or silo sugar storage [2].

To better understand the loose and moist aggregate behaviour, it is useful to measure the multi-axial shearing response of dry and moist sugar granules. The present study is therefore an investigation of the shearing response of a loose, dry or moist, sugar aggregate mainly by triaxial tests, as typically used in soil mechanics [3]. Its originality lies in two main points: firstly, we use original test conditions. The aggregates are loose, dry or moist, and tested up to about 20\% axial strain without any noticeable barrelling of the specimen; sugar is subjected to low confining pressures (from 15 to $90 \mathrm{kPa}$ ) which are assumed representative of the industrial loading conditions. As a matter of comparison, Kolymbas et al. [2] performed triaxial tests on a dry sugar aggregate up to $10 \%$ axial strain for confining pressures ranging from $50 \mathrm{kPa}, 100 \mathrm{kPa}$ and up to $800 \mathrm{kPa}$; Kamath et al. [4] tested dry sugar up to $15 \%$ axial strain under three low pressure values, see Table 1. Our experimental procedure is also adapted to testing an aggregate which is highly soluble in water. Heavily lubricated specimen ends avoid frictional end effects and, 
in combination with a 1:1 sample elongation, potential buckling is also avoided. Secondly, our interpretation of test results is based on Rowe's theory [5] of frictional and dilatant shear behaviour. Hence critical states of shear flow at constant volume are better characterised from raw test results, and parameters representative of the sugar aggregate are identified for use in sugar flow and yield modelling.

In practice, a circular cylindrical sample of sugar is subjected to an arbitrary combination of a confining pressure $P$ superimposed on a uniaxial stress $\sigma_{a}$, see Fig. 1(a). To follow convention within the soil mechanics literature, we assume that the applied axial stress $\sigma_{a}$ and confining pressure $P$ are positive in compression. (Unless otherwise stated all stress quantities are true Cauchy values.) Sugar crystals are either dry or mixed either with sugar-saturated water (to mimic molasses in industrial refinement) or with glycerol, which has a low solubility for sugar but a similar viscosity to that of molasses at the industrial processing temperature of $70^{\circ} \mathrm{C}$. Our tests are referred to as drained triaxial tests in soil mechanics: the sugar aggregate is either dry or is moistened by a small amount of fully-saturated aqueous sugar solution or glycerol; no pore pressure effects could be expected beyond some small capillary pressures at grains contact. The measured stresses on the sample are 'effective stresses' in soil mechanics parlance' ${ }^{1}$. Before any barrelling of the specimen occurs, the principal stresses are assumed uniform inside the sugar sample and have the following expressions:

$$
\begin{gathered}
\sigma_{1}=\sigma_{a}+P \\
\sigma_{2}=P \\
\sigma_{3}=P
\end{gathered}
$$

At low strain rates (less than about $10^{-5} s^{-1}$ ) liquid-assisted creep of sugar is observed [6]. The focus of the present investigation is at much higher strain rates (exceeding $10^{-3} s^{-1}$ )

\footnotetext{
${ }^{1}$ In the following, all stresses referred to are effective stresses, hence no dash is used to distinguish them.
} 
where the powder aggregate shears by a combination of sliding and rolling at the elastic frictional contacts between particles, as will be shown (see subsection 3.2). In the following, the triaxial test arrangement is described, and the test reproducibility is investigated. To that purpose, we analyse the effect of limited variations in the initial loose sample density upon the stress-strain response. The degree of damage to the crystals during triaxial testing is determined by post examination using the scanning electron microscope and by comparing grain size distributions. Results for dry and wet sugars are reported, and the initial normalised Young's modulus is identified. Rowe's stress-dilatancy theory is then used to organise the data of triaxial shearing and to derive two parameters representative of the sugar aggregate: firstly, the stress ratio at zero dilatancy-rate $K_{\mu}$, and secondly the critical state stress ratio $K_{c v}$ at which the aggregate of grains could participate in frictional flow at constant volume. Additionally, the influence of cohesion in the moist aggregate is examined. The sensitivity of the aggregate triaxial response to the strain rate, particle size, and type of liquid mixed with crystals are each investigated according to their relative influence on the initial Young's modulus and stress-dilatancy results.

\section{Experimental method}

\section{$2.1 \quad$ Test device}

A new triaxial test rig was designed to measure the multi-axial shearing response of loose sugar aggregates to superimposed deviatoric and hydrostatic stress, see Fig. 1(b). The rig is based on the conventional "triaxial cell" used in soil mechanics [3], but has been adapted to deal with the case of low confining pressures on the order of 10 to $100 \mathrm{kPa}$ which are of the order of magnitude of those encountered in industrial applications. 


\subsubsection{Testing procedure}

The principle of operation of the triaxial test rig is as follows. A 1:1 circular cylindrical sample of sugar (50mm diameter by $50 \mathrm{~mm}$ height) was placed inside a latex sleeve, and between lubricated ends. Two latex discs covered with $2 \mathrm{~mm}$ thick PTFE grease were inserted at each end of the specimen, so as to minimise friction with the loading platens. The sample preparation was achieved by initially wrapping the sleeve around a split metal mould, and then hand spooning in the sugar as recommended by ASTM standard [7]. This produced a relatively loose structure (lower density samples). Whenever a denser packing was needed, each spoonful of sugar was stirred into place (higher density samples). By doing so, the sugar initial density was slightly varied at the lower end of the possible range (see subsection 2.2.3). The sleeve was then sealed and a constant confining pressure $P$ of up to $95 \mathrm{kPa}$ was imposed by the atmosphere external to the sleeve. Only then was the split metal mould removed, just before the test began.

The confining pressure $P$ was due to partial air evacuation of the latex sleeve, using a vacuum pump ${ }^{2}$ connected to the bottom of the specimen via a porous disk and drilled hole within the lower loading platen. Rejected tests were those for which PTFE grease blocked the porous disk, hence hindering the application of the confining pressure $P$ to the sugar. The specimen was loaded additionally by a compressive stress $\sigma_{a}$ via the upper loading platen fastened to a screw-driven test machine. The test procedure consisted of first setting the desired confining pressure, and then applying an axial contraction at a fixed displacement rate $\dot{d}$ with the confining pressure held constant. The imposed axial strain rate $\dot{\varepsilon_{a}}$ was either $0.16 \%$ sec (low) or $3.3 \% /$ sec (high), corresponding to cross-head displacement speeds of 5 or $100 \mathrm{~mm} / \mathrm{min}$. The lower strain rate is on the order of those used in $[2,4]$, see Table 1 . The higher strain rate is comparable to that encountered within

\footnotetext{
${ }^{2}$ The vacuum pump is of model type NSE800, and was manufactured by KNF Neuberger. It features a gas separator and a condenser to separate out vapours; The vacuum pump has a feedback controller (model type NC800) to maintain the pressure to any desired value.
} 
an industrial centrifuge.

\subsubsection{Measurement devices and accuracy}

A load cell was attached to the screw-driven testing machine. Its capacity is $5 \mathrm{kN}$ and it measures to an accuracy of $\pm 0.5 \mathrm{~N}$. The gauge pressure $P$ at the bottom of the sample was measured to an accuracy of $\pm 0.5 \mathrm{kPa}$ by a silicon diaphragm pressure transducer placed between the sample and the vacuum pump. A reflective laser extensometer measured the axial spacing $l$ between two marker lines adhered to the latex sleeve over an initial gauge length $l_{0}$ of $12 \mathrm{~mm}$ (about one-fifth of the sample height); the measurement accuracy is $\pm 10 \mu \mathrm{m}$. An emissive-receptive laser measured the diameter $d$ at mid-length of the specimen to an accuracy of $\pm 10 \mu \mathrm{m}$, see Fig. 1(b). The first measurement gave the true axial strain $\varepsilon_{a}=-\log \left(l / l_{0}\right)$. The sugar specimen was assumed to remain a circular cylinder in its central zone throughout the test viz. up to 30 to $35 \%$ axial strain according to visual inspection. Hence the two lasers were used to evaluate the true volumetric strain

$\varepsilon_{V}$ in the central zone: $\varepsilon_{V}=-\log \left(V / V_{0}\right)$ where $V=\pi / 4 d^{2} l$ is the current central volume and $V_{0}$ is its initial value. Following soil mechanics convention, all strains $\varepsilon_{a}$ and $\varepsilon_{V}$ were taken positive in compression.

One should note that volumetric strains are measured more directly in the triaxial testing of sands, for example, by ensuring that samples are saturated with water prior to testing, and by accurately recording inflow or outflow of pore water during a test. In the present case, sugar being soluble in water and many other liquids, the direct approach could not be followed. Instead, a partial vacuum was applied to the aggregate. The use of a laser to record changes of length of a single diameter is bound to lead to greater error in estimating volumetric strain, especially if the sample suffers barrelling or strain localisation beyond peak strength. In subsection 3.6, we use Rowe's theory to assess the beginning of strain localisation more accurately than could be achieved simply by visual inspection. 


\subsection{Physical characteristics and preparation of the sugar aggre- gates}

Unless otherwise stated, all tests were performed using extra fine white sugar (reference code 55217) supplied by British Sugar Plc, Ipswich. Several triaxial tests were also performed on dry granulated cane sugar from Tate \& Lyle. Both aggregates are made of uniformly graded crystalline sucrose $\left(\mathrm{C}_{12} \mathrm{H}_{22} \mathrm{O}_{11}\right)$, as shown in the SEM micrograph of Fig. 2(a). Sucrose crystals have the form of elongated anhydrous monoclinic prisms [8]. The moisture content of the as-received sugar was measured by evaporation of the dissolved water in an oven at $140^{\circ} \mathrm{C}$ for 50 hours; measurement of mass loss indicates a water content only on the order of 0.1 mass $\%$ for both types of sugar.

\subsubsection{Grain size distributions}

For both sugars, the grain size distribution was evaluated from 10 samples (average mass 80 grams each) each poured from a test tube through an Accusizer laser sensor ${ }^{3}$; each sample was made using a rotating riffler which uniformly distributed sugar from a large funnel to the 10 test tubes.

In the case of British Sugar sugar, Fig. 2(b) shows that prior to testing, the crystals have a narrow size distribution; their average diameter $d_{50}$ (corresponding to $50 \%$ of the grains) is $440 \mu \mathrm{m} \pm 40 \mu \mathrm{m}$ and the uniformity coefficient $C U=d_{60} / d_{10}=1.8$ where $d_{10}$ (resp. $d_{60}$ ) is the diameter corresponding to $10 \%$ (resp. 60 \%) grains passing through the sieves. Other sugar aggregates found in the literature $[2,4]$ have a similar mean grain size to that of British Sugar sugar, see Table 1. Its grain size characteristics also approach those of Hostun sand $\left(d_{50}=350 \mu \mathrm{m}\right.$ and $C U=1.7$, see [9] and [10]), and Karlsruhe medium sand $\left(d_{50}=330 \mu \mathrm{m}\right.$ and $C U=1.9$, see [11], [2]). Furthermore, the optical sizing

\footnotetext{
${ }^{3}$ The Accusizer laser sensor was manufactured by Particle Sizing Systems, Santa Barbara, California, USA.
} 
technique was compared with sieving [12] on an average of 4 samples of average mass $200 \mathrm{~g}$ each, see Fig. 2(b). The particle size distribution is globally shifted to lower values (with an average diameter of $400 \mu \mathrm{m} \pm 2 \mu \mathrm{m}$ ) but remains within the range obtained with the optical technique. This small difference in average size is explained as follows: whereas the laser sensor measures the grain size in a random direction (tridimensional measurement), grains pass through the sieves in the direction of their smallest dimension (unidimensional measurement) [13]. As sucrose crystals are elongated (viz. far from spherical), this implies a generally smaller grain size distribution observed with sieving. Nevertheless, both techniques give similarly shaped grain size distributions and a limited difference of about $10 \%$ in the average grain diameter evaluation.

The grain size distribution of Tate \& Lyle sugar is broader than that of British Sugar sugar, with a $C U=2.2$, see Fig. 2(b). This characteristic is close to that of Chattahoochee river sand $(C U=2.24)$ and Berlin sand $(C U=2.27)$ [11]. Its average grain size is of $810 \mu \mathrm{m} \pm 40 \mu \mathrm{m}$ which is about twice that of British Sugar sugar.

\subsubsection{Wet sample preparation}

Wet British Sugar sugar was prepared by hand-mixing sugar crystals with a given amount of sugar-saturated water, obtained as follows. Full saturation was ensured by heating the water to $70^{\circ} \mathrm{C}$ before dissolving sugar to saturation. The syrup was then cooled to ambient temperature $\left(20^{\circ} \mathrm{C}\right)$. Its density is $\rho_{\text {sat }}=1.30 \pm 0.01 \mathrm{Mg} / \mathrm{m}^{3}$. Sugar crystals and syrup were then mixed so that the syrup represented $10 \%$ of the total sample by mass. The sugar mix was used immediately after preparation, as sugar re-crystallises due to supersaturation of the cooled solution. The triaxial response of sugar wetted with glycerol was also explored. Glycerol has a viscosity similar to that of molasses in massecuite at typical processing temperatures (about $70^{\circ} \mathrm{C}$ ), with the added advantage that glycerol does not dissolve sugar crystals. Its density is similar to that of sugar-saturated water: 
$\rho_{\text {glycerol }}=1.26 \pm 0.01 \mathrm{Mg} / \mathrm{m}^{3}$. The preparation of sugar wetted with glycerol was identical to that of sugar wetted with sugar-saturated water.

\subsubsection{Samples initial density}

The initial unit weight of each dry or wet sugar specimen is given by:

$$
\gamma_{0}=(\text { mass } m \text { of dry grains } \times g) /\left(\pi d_{0}^{2} l_{0} / 4\right)
$$

where $m$ is the mass of dry aggregate ${ }^{4}$, measured with an accuracy of 0.01 grams, $g$ is gravity $\left(9.81 \mathrm{~m} . \mathrm{sec}^{-2}\right), d_{0}$ is the initial diameter of the sample (measured with the laser device, see subsection 2.1.2) and $l_{0}$ is the initial sample length, taken as $50 \mathrm{~mm}: l_{0}$ was assumed equal to the split mould length as the mould was removed only after the confining pressure $P$ had been applied to the sample. For all of the 54 tests carried out, $\gamma_{0}$ varied from $7.25 \mathrm{kN} / \mathrm{m}^{3}$ to $9.74 \mathrm{kN} / \mathrm{m}^{3}$. Using $\gamma_{S}=15.56 \mathrm{kN} / \mathrm{m}^{3}$ [8], this corresponds to initial void $\operatorname{ratios}^{5} e_{0 \text { min }}=0.60$ and $e_{0 \max }=1.15$. As a matter of comparison, see Table 1 , tests on loose sugar by Kolymbas et al. [2] had similar initial densities: $\gamma_{0}$ varied from 8.46 to $9.49 \mathrm{kN} / \mathrm{m}^{3}$, implying $e_{0 \min }=0.64$ and $e_{0 \max }=0.84$. Kamath et al. [4] only provided their average initial density $\gamma_{0 \text { mean }}=9.04 \mathrm{kN} / \mathrm{m}^{3}$ which is $7 \%$ higher than the average in the present study.

\section{Results and discussion}

The triaxial compressive response of dry and wet sugars was determined at an ambient temperature of $20^{\circ} \mathrm{C}$. The reproducibility of the tests is first investigated, and then the

\footnotetext{
${ }^{4}$ In the case of wet aggregates, the liquid mass is substracted from the total sample mass $m_{T}$ using that it represents $10 \% m_{T}$.

${ }^{5}$ Initial void ratio $e_{0}$ is defined as the ratio of the volume of void space to the volume of solid particles and is also written as: $e_{0}=\left(\gamma_{S} / \gamma_{0}\right)-1$.
} 
level of damage to the grains. Tests have been conducted to explore the effect of strain rate, sugar grain size and addition of liquid upon the initial Young's modulus and stressdilatancy response. The influence of cohesion in moist aggregates is also examined. Finally, comparison of critical state friction angles with traditional soils is proposed.

\subsection{Reproducibility of the tests}

At each of three given confining pressures $P=22,52$ or $75 \mathrm{kPa}, \gamma_{0}$ is varied from a lower value range $\left(8.10 \leq \gamma_{0} \leq 8.40 \mathrm{kN} / \mathrm{m}^{3}\right)$ to a higher value range $\left(8.95 \leq \gamma_{0} \leq 9.74 \mathrm{kN} / \mathrm{m}^{3}\right)$. The corresponding axial stress-axial strain curves are plotted, see Fig. 3. At each given pressure, the maximum semi-difference in axial stress between stress-strain curves in the two density ranges is only $0.5 \%$ of the average of the curves and this occurs at $0.1 \%$ axial strain (very beginning of the test): above $0.1 \%$ axial strain and all along the test, the semidifference is under $0.5 \%$. Therefore within the range tested (here $8.10 \leq \gamma_{0} \leq 9.74 \mathrm{kN} / \mathrm{m}^{3}$ ) the effect of initial density upon stress data is considered negligible. This also assesses a good reproducibility of the stress-strain data at each given confining pressure. Nevertheless, volumetric strain versus axial strain curves are much less reproducible than stress-strain curves, see Fig. 3. This feature is also noticed by [14] in the case of drained triaxial tests on loose Hostun sand $(P=100$ and $300 \mathrm{kPa})$, some tests being even only slightly dilatant, others exhibiting negligible volume variations.

\subsection{Examination of sugar crystals after triaxial testing}

The grain size distribution of dry sugar has also been evaluated after triaxial compression at a high confining pressure (within the range tested) and at the highest strain rate available $\left(P=80 \mathrm{kPa}\right.$ and $\left.\dot{\varepsilon_{a}}=3.3 \% / \mathrm{sec}\right)$ by a traditional sieving technique, see Fig. $2(\mathrm{~d})$. Measurements have been conducted on three samples of average mass $200 \mathrm{~g}$ each. The 
particle size distribution is not noticeably affected by triaxial testing. This is confirmed by SEM photographs taken after the test, Fig. 2(c): they exhibit no major difference with photographs taken before the tests, see for example Fig. 2(a). It is concluded that the sugar crystals deform as elastic, frictional particles with neither plastic deformation nor crushing at particle contacts.

\subsection{Results of triaxial compression for dry and wet sugars}

Figs. 4 and 5 show typical test results obtained on dry and wet British Sugar sugars. Similar results are obtained with Tate \& Lyle sugar. Stresses have been normalised via the principal stress ratio $K=\sigma_{1} / \sigma_{3}$ and axial strains are plotted up to $35 \%$. For a granular material with an angle of internal friction $\Phi, K=(1+\sin \Phi) /(1-\sin \Phi)$, see for example [12].

On all stress-strain plots, a stable hardening behaviour is observed (no peak stress) and the stress data tend to a horizontal asymptote relatively independent of confining pressure and initial density. Indeed Kolymbas et al. [2] show a noticeable dependence of stressstrain curve asymptotes to confining pressure from $P=100 \mathrm{kPa}$ only. This phenomenon is explained more accurately in subsection 3.6.4 as the consequence of a greater aggregate instability with increasing $P$.

When considering the evolution of volumetric strain versus axial strain, an initial compressive stage is observed, followed by gradual dilatation of the specimen. Nevertheless, the end of test, corresponding to the beginning of strain localisation, is difficult to deduce from the sole curves of Figs. 4 and 5. Hence although one can observe one (or more) phases of dilation up to a phase of flow at constant volume- a critical state, it is not possible to know from Figs. 4 and 5 whether this critical state is reached towards the end of test or not. This aspect is dealt with in the forthcoming subsections with the help of Rowe's theory. As already mentioned in subsection 3.1, volumetric strain evolution with axial strain 
displays noticeable variability, contrarily to stress-strain curves. These results are typical of those obtained for loose samples of sand, e.g. Hostun sand [14]. Evaluating $\varepsilon_{V}$ from a single sample diameter adds to the scatter, especially where the deviator stress is reaching its ultimate value. Nevertheless, several aggregate properties are deduced from these data, as shown in subsection 3.6.

\subsection{Initial Young's modulus}

The initial normalised Young's modulus $\frac{E_{0}}{\sigma_{3}}$ is determined for all tests as the slope of the best linear fit in a least squares sense for data points $\left(\varepsilon_{a}, K\right)$ and for strains smaller than $0.2 \%$, see Figure 6(a). With the added advantage of using experimental data directly, this method is close to that of Kolymbas et al. [2] who define $E_{0}$ as $\left(\left(\dot{\sigma_{1}}-\dot{\sigma_{3}}\right) / \dot{\varepsilon}_{1}\right)_{\varepsilon_{1}}=0$. In this formula $\dot{\sigma_{1}}\left(\right.$ resp. $\dot{\sigma_{3}}$ and $\dot{\varepsilon_{1}}$ ) are evaluated from $\sigma_{1}(t)$ (resp. $\sigma_{3}(t)$ and $\left.\varepsilon_{1}(t)\right)$ at each timestep $t$ as the slope of the least mean square linear interpolation of the four neighbouring points to $t$. Furthermore, using our method, all test data display good linear correlation with Pearson's coefficient of correlation $r^{2}$ [15] varying from 0.54 to 0.82 .

Figs. 6(b), (c) and (d) display the evolution of $\frac{E_{0}}{\sigma_{3}}$ versus confining pressure $P$ for the five types of test performed. A linear fit was performed in a least squares sense between $\frac{E_{0}}{\sigma_{3}}$ and $P$ : an acceptable linear correlation is observed for all tests on dry sugars, see Table 2 . Tests on wet sugars exhibit insufficient $r^{2}$ viz. $r^{2}$ below $50 \%$. Additionally, our results are compared to those proposed in [2] on sugar and loose or dense Karlsruhe sand.

Results at low strain rate are in good agreement with [2] on loose sugar, see Fig. 6(b). Moreover, Table 2 shows a noticeable effect of strain rate upon the slope of the linear fit which is divided by 4 when strain rate is multiplied by 20. Grain size effect is also significant when evaluated at high strain rate, see Fig. 6(c) and Table 2: the vertical intercept of $\frac{E_{0}}{\sigma_{3}}$ versus $P$ linear fit is increased by a factor of 2 when mean grain size is multiplied by 2 , and the associated slope is also increased by a factor of 3 . The difference is so great that where 
British Sugar sugar behaviour can be compared to that of loose Karlsruhe sand at low strain rate, see Fig. 6(c), Tate \& Lyle sugar behaviour is closer to that of dense Karlsruhe sand. However, the addition of $10 \%$ moisture (either sugar saturated water or glycerol) at

high strain rate has no noticeable effect upon $\frac{E_{0}}{\sigma_{3}}$ versus $P$ linear fit, see Fig. 6(d). No linear fit represents better data for wet sugars than that identified for dry sugar at an identical strain rate.

\subsection{Critical state}

As mentioned above (see subsection 3.3), it is not easy to determine whether a critical state is reached before the end of test from Figs. 4 and 5 only. Indeed data plotted up to $35 \%$ axial strain do not obviously display all the features indicating that a critical state is reached [16]: although stress ratio rapidly approaches an asymptote from 10 to $20 \%$ axial strain, volumetric strain does not obviously and definitely reach a plateau, except for a few tests (e.g. test $\mathrm{n}^{\circ} 1$ for dry sugar at $\dot{\varepsilon}_{a}=0.16 \% / \mathrm{sec}$ ). In order to overcome this difficulty and accurately characterise critical state for each test, we propose the following interpretation due to Rowe [5]. This also brings more insights into the aggregate behaviour during the triaxial test.

\subsection{A stress-dilatancy interpretation}

Rowe [5] proposed a "stress-dilatancy" theory for the shearing of a granular aggregate accounting for interlocking of grains on what would, in a continuum, have been the preferred plane of sliding.

Three regimes are described for the triaxial compressive testing of an aggregate [5], see Fig. 7(a). At low stress ratios (here designed regime $\alpha$ ), particles rearrange themselves in an unorganised manner attaining a denser packing. From that stage, particles can move no 
more unless by overriding one of their neighbouring particles: at a macroscopic level, the aggregate tends to dilate (regime $\beta$ ). During the $\beta$-regime, Rowe [5] relates the principal stress ratio $K=\frac{\sigma_{1}}{\sigma_{3}}$ to the rate of dilatancy $\frac{d \varepsilon_{v}}{d \varepsilon_{a}}$ :

$$
K=K_{r}\left(-\frac{d \varepsilon_{v}}{d \varepsilon_{a}}\right)+K_{\mu}
$$

The stress ratio $K$ can also be related to the angle of internal friction $\Phi$ mobilised on the planes of maximum stress obliquity, derived by dropping tangents from the origin on to a Mohr circle of stress. The mobilised stress ratio $K=K_{\mu}$ which is linked via Eqn. 1 with zero dilatancy-rate in the $\beta$-regime also corresponds, in Rowe's theory, with the mobilisation of $\Phi=\Phi_{\mu}$ within the aggregate, where $\Phi_{\mu}$ is the inter-particle angle of friction. The third regime $\gamma$ occurs after the point of peak dilatancy-rate which is generally found to correspond to the point of peak stress ratio. This softening tends to lead to localisation, with shear bands forming at angles of between $\left(45^{\circ}+\Phi_{\max } / 2\right)$ and $45^{\circ}$ to the horizontal plane of maximum compressive stress. The ultimate condition of shear at constant volume, also called a critical state, is characterised by a critical stress ratio $K_{c v}$, or a corresponding angle of friction $\Phi_{c v}$. Typically, $\Phi_{c v}$ is found to exceed the inter-particle angle of friction $\Phi_{\mu}$ by $5^{\circ}$ to $10^{\circ}$ in sands. Rowe [5] recognised that the ultimate mobilisation of $\Phi_{c v}$ at zero dilatancy-rate provided a challenge to the generality of Eqn. 1. He attempted to resolve the apparent conflict by using a micro-mechanical argument, proposing that the angles of contact-planes between particles would be narrowly distributed in the $\beta$-regime, but much more widely distributed in the more chaotic $\gamma$-regime. Whereas the average dilatancy-rate is zero at a critical state, it might be argued that the contact forces are not uniformly distributed between "weak" contacts offering local contraction and "strong" contacts offering local dilation. The latter might account for the bulk of the stresses delivered through contacts leading to the $5^{\circ}$ to $10^{\circ}$ difference between $\Phi_{\mu}$ and $\Phi_{c v}$. This 
difference is therefore understood as being due to local dilation in an aggregate that, on average, is no longer changing volume. Not withstanding the semi-empirical nature of Rowe's stress-dilatancy theory, there is a great deal of evidence that the concepts of critical state stress ratios, enhanced by dilatancy ratios broadly in the fashion of Rowe, are a usual framework in which to place the shearing of sands [11], as well as other granular materials [5]. A similar approach will therefore be adopted here.

Carefully characterising the three regimes (and the associated parameters $K_{r}, K_{\mu}$ and $K_{c v}$ ) for each test allows a critical state to be defined as the moment when stress ratio $K_{c v}$ is reached. Assuming $\left(\frac{d \varepsilon_{v}}{d \varepsilon_{a}}\right)$ is known, it is the moment (or equivalently the axial strain $\varepsilon_{a}$, $\varepsilon_{a}$ being a monotonic function of time-step $t$ ) when $\left(\frac{d \varepsilon_{v}}{d \varepsilon_{a}}\right)$ reaches zero for the second time during the test, the first time corresponding to $K_{\mu}$, see Fig. 8. In the absence of localisation, $\left(\frac{d \varepsilon_{v}}{d \varepsilon_{a}}\right)$ would be expected to approach zero asymptotically. In real triaxial tests, the approach to a critical state will be erratic, especially as $3 \mathrm{D}$ behaviour is viewed by a pair of lasers inspecting only two gauge lengths. Fig. 8 indicates the judgment that is called for in the selection of an axial strain $\varepsilon_{a c v}$ at which the dilatancy-rate reaches zero for the first time after the linear $\beta$-regime. Beyond $\varepsilon_{a c v}$ the measurements of strain become systematically erratic, possibly due to the onset of barrelling and/or strain localisation, and the data must therefore be discarded. This point has been delayed as far as $\varepsilon_{a}=20 \%$, as in the example shown in Fig. 8, by the use of friction-reduction on the platens, and by the selection of a 1:1 aspect ratio for the sample which militates against the formation of shear bands steeper than $45^{\circ}$. Furthermore, for all five type of tests, it is shown that $\varepsilon_{\text {acv }}$ varies hugely, from 9 to $30 \%$ with a median value of $20 \%$ (viz. most common value). Nevertheless and most interestingly $\varepsilon_{a c v}$ corresponds to a plateau of the $\left(\varepsilon_{a}, \varepsilon_{v}\right)$ curve: a close examination shows that for each test, reaching $\varepsilon_{a c v}$ always corresponds to the beginning a small plateau (width of a few percent axial strain only) which was not obvious to spot on plots going up to $35 \%$ axial strain. A good example is test $\mathrm{n}^{\circ} 2$ at $\dot{\varepsilon_{a}}=0.16 \% / \mathrm{sec}$, see Fig. 4(a): 
$\varepsilon_{a c v}=25 \%$ marks the beginning of a $2 \%$ axial strain wide plateau for the $\left(\varepsilon_{a}, \varepsilon_{v}\right)$ curve after which the dilatancy-rate (not shwon) moves away from zero and becomes erratic, therefore marking the end of test. In brief, the characterisation of critical state and end of test is easier when using stress/dilatancy-rate plots, as first proposed by Rowe [5], rather than just stress-strain and $\left(\varepsilon_{a}, \varepsilon_{v}\right)$ curves.

\subsubsection{Determination of the parameters}

The application of Rowe's theory requires the calculation of the rate of dilatancy $\left(\frac{d \varepsilon_{v}}{d \varepsilon_{a}}\right)$. It is derived from data $\varepsilon_{v}(t)$ and $\varepsilon_{a}(t)$ as follows. Firstly, $\varepsilon_{v}(t)$ and $\varepsilon_{a}(t)$ are filtered to limit noise on $\left(\frac{d \varepsilon_{v}}{d \varepsilon_{a}}\right): \varepsilon_{v}(t)$ and $\varepsilon_{a}(t)$ are averaged over 21 data points, where each test comprises about 500 data points, see Fig. 7(b). The number of filtering points (i.e. 21) was chosen so that the shape of initial data $\varepsilon_{v}(t)$ and $\varepsilon_{a}(t)$ are respected but filtered as shown in Fig.7(b). It is noted that $\varepsilon_{a}$ is not a linear function of time $t$ up to about $8 \%$ axial strain although $\dot{d}$ (crosshead displacement speed) is held constant. This is due to the compression of the greasy latex discs at each end of the specimen which, of course, does not affect the measurement of $\varepsilon_{a}$ over the gauge length. Nevertheless this strain-rate distorsion is too small to affect judgments about rate effects (see subsection 3.6.2). Finally $\left(\frac{d \varepsilon_{v}}{d \varepsilon_{a}}\right)$ at timestep $t$ is equal to ratio $a_{\text {volumetric }}(t) / a_{\text {axial }}(t)$, where $a_{\text {volumetric }}(t)$ (resp. $\left.a_{\text {axial }}(t)\right)$ is the slope of the linear fit (in a least squares sense) to volumetric strain (resp. axial strain) versus time at $(t-2),(t-1), t,(t+1)$ and $(t+2)$. This technique is similar to that suggested in [2]. A typical plot giving $K=\sigma_{1} / \sigma_{3}$ versus $\left(\frac{d \varepsilon_{v}}{d \varepsilon_{a}}\right)$ is shown in Fig. $7(\mathrm{c}) . K_{r}$ and $K_{\mu}$ are directly derived from this plot as the parameters of the linear fit between $K$ and $\left(\frac{d \varepsilon_{v}}{d \varepsilon_{a}}\right)$ after the disorganised compaction regime corresponding to low stress ratios (regime $\alpha$ ). $K_{c v}$ is rather derived from Fig. 8, as explained above.

The foregoing interpretations lead to the summary of the stress-dilatancy which was typified for a particular test on sugar in Fig. 7(a) and (c). There is a striking difference 
in behaviour from that reported for sands [5]. Although the maximum rate of dilatancy $\left(-\frac{d \varepsilon_{v}}{d \varepsilon_{a}}\right)_{\max }=0.9$ mobilised at the end of the $\beta$-regime was similar to that experienced in dense sands, there was no reduction in stress ratio experienced in the subsequent $\gamma$-regime. Instead, the dilatancy-rate dropped progressively from its peak value to zero while the stress ratio remained effectively constant. These differences presumably arise from the blocky shapes of sugar grains in contrast with typically-rounded sand grains. It must certainly be expected that the range of void ratios available to blocky grains would be much wider than the range enjoyed by rounded grains. Stirring, or tamping, would still leave blocky grains relatively disordered, and it is easy to understand how the mobilisation of small shear strains in the $\alpha$-regime could lead to local alignments and densification. At larger shear strains, the opposite effect would be bound to occur, leading to substantial dilatancy in the $\beta$-regime. However, once that dilatancy has opened up the fabric sufficiently, by $5 \%$ in Fig. 7(b), it can be argued that the blocky grains would then simply tumble over each over in shearing at a critical state. Sands, on the other hand, typically reach their peak dilatancy rate while still at, or close to, their original density. In sands, the increase in void ratio to its critical value takes place after peak strength has been achieved whereas for the blockier sugar grains in these tests, that increase has apparently already taken place prior to peak strength. It is likely that stress ratios greater than $K_{c v}$ might have been observed if the initial density of the grains had exceeded the maximum value of $9.74 \mathrm{kN} / \mathrm{m}^{3}$ applicable in the current tests. It is likely that the blocky grains shown in Fig. 2 could have been packed at a void ratio as low as 0.2 , corresponding to $\gamma_{0}=13 \mathrm{kN} / \mathrm{m}^{3}$, if dry crystals had been gently vibrated after placement. From this perspective, the unusual nature of the shearing of sugar crystals, compared with rounded sand grains, is that relatively loose packings display overall dilatancy without correspondingly high stress ratios. 


\subsubsection{Effect of strain rate, grain size and moisture upon $K_{\mu}$ and $K_{c v}$}

Mean values and coefficients of variation for parameters $K_{\mu}$ and $K_{c v}$ are given in Table 3 for each of the five types of tests performed. $K_{r}$ values are not reported because, contrarily to $K_{\mu}$ and $K_{c v}$, this parameter varies tremendously, from 0.3 to 3 . Indeed $K_{r}$ represents the transient rearrangement of the granular fabric, and especially its tendency to contract or dilate. Rowe's theory specifies that the rearrangement mechanism would be sliding (without rotation) at points of contact, and it predicted $K_{r}=K_{\mu}$. Latest evidence from DEM [17] shows that grain rotation is much more in evidence. The rate of volume change during phase denoted $(\beta)$ must be strongly influenced by the tendency of these blocky grains to aggregate or dis-aggregate. It is therefore not surprising that $K_{r}$ is a strong function of strain rate, fluid and pressure (representing the nature of contact forces). On the contrary, $K_{\mu}$ and $K_{c v}$ are independent of $P$ and initial density: their coefficient of variation is low for each type of tests performed, see Table 3. In order to evaluate their dependence upon strain rate, grain size and moisture, a statistical test was performed as follows, see Figs. 9. For two given test conditions (e.g. dry sugar or sugar + glycerol), the difference between mean $K_{\mu}$ is considered statistically significant if there is no overlap between the intervals of confidence $\left[K_{\mu}-2 \operatorname{std}\left(K_{\mu}\right) ; K_{\mu}+2 \operatorname{std}\left(K_{\mu}\right)\right]$, where $\operatorname{std}\left(K_{\mu}\right)$ is $K_{\mu}$ standard deviation. Let us assume that the data distribution is Gaussian for each type of test. Then $95 \%$ of $K_{\mu}$ data are in the interval of confidence $\left[K_{\mu}-2 \operatorname{std}\left(K_{\mu}\right) ; K_{\mu}+2\right.$ $\left.\operatorname{std}\left(K_{\mu}\right)\right][18]$. Hence if there is no overlap between the two intervals of confidence, the difference between the two mean values of $K_{\mu}$ is considered statistically significant. The same statistical test is applied with $K_{c v}$. A less restrictive test consists in considering the intervals of confidence $\left[K_{\mu^{-}} \operatorname{std}\left(K_{\mu}\right) ; K_{\mu}+\operatorname{std}\left(K_{\mu}\right)\right]$, see Figs. 9, but in that case only $70 \%$ of $K_{\mu}$ data are in the interval of confidence. It is shown that this second test gives identical results for $K_{\mu}$ and $K_{c v}$ comparisons. Indeed both tests show that there is no statistically significant difference between low and high strain rate $K_{\mu}$ and $K_{c v}$, neither between British 
Sugar and Tate \& Lyle sugar $K_{\mu}$ and $K_{c v}$, nor between dry sugar and sugar $+10 \%$ glycerol $K_{\mu}$ and $K_{c v}$, see Figs. 9(a), (b) and (c). There is only a statistically significant difference in $K_{c v}$ between dry sugar and sugar $+10 \%$ sugar saturated water. In brief, there is no effect upon $K_{\mu}$ and $K_{c v}$ of strain rate, mean grain size or of the addition of $10 \%$ glycerol, see Fig. 9(d). There is only an effect of the addition of $10 \%$ sugar saturated water on $K_{c v}$. Hence $K_{\mu}$ and $K_{c v}$ appear to be intrinsic parameters to sugar, except that the addition of $10 \%$ sugar saturated water appears to be capable of reducing $K_{c v}$ towards the value of $K_{\mu}$.

\subsubsection{Friction and cohesion}

It is well understood that an aggregate of moist grains will exhibit cohesion due to the additional intergranular normal contact forces induced by the capillary suction of fluid droplets. The capillary pressure $P_{c}$ of a water-saturated granular medium with pore openings of diameter $d_{p}$ would be $4 T / d_{p}$, where $T$ is the surface tension of the fluid which

presumed to wet the grains. For water at $20^{\circ} \mathrm{C}, T \approx 6 \times 10^{-5} \mathrm{kN} / \mathrm{m}$, and for $d_{p}=0.3 \mathrm{~mm}$ corresponding to the $10 \%$ tails of the cumulative frequency distribution of particle sizes in Fig. $2(\mathrm{~b}), P_{c} \approx 0.8 \mathrm{kPa}$. The fluid content required to saturate sugar grains at a typical void ratio of 0.75 is about $47 \%$ by mass, so the addition of only $10 \%$ fluid will create more tightly curved menisci than those associated with saturated pores. Nevertheless, the capillary effect is considered to be small in relation to applied suction $P$ in the range 15 to $95 \mathrm{kPa}$. This tends to be borne out by values in Table 3 for $\Phi_{c v}$ and $\Phi_{\mu}$, interpreted without reference to $P_{c}$ in the case of moist sugar, which are reasonably consistent throughout. If sugar were to be sheared at water contents much lower than $10 \%$, additional capillary effects would have to be considered. 


\subsubsection{Comparison with various aggregates}

For dry sugar at low strain rate, $\varphi_{c v}$ is evaluated at $36.3^{\circ}$ and it is independent of confining pressure. This result is confirmed by Kolymbas et al. [2] who identify an identical value of $36^{\circ}$ in similar conditions $(P=50 \mathrm{kPa})$, although using a different yield criterion than ours, see Table 1. Nevertheless Kolymbas et al. [2] show that $\varphi_{c v}$ decreases for $P$ greater than $100 \mathrm{kPa}$. This is explained as the consequence of a more unstable packing of grains before critical state, due to the diminished possibility for grains to rearrange in the $\alpha$ and $\beta$-regimes with increasing $P$. Our results are also in broad agreement with Kamath et al. [4]: a limit state friction coefficient of $35^{\circ}$ is identified although for a different sugar and using a different yield criterion, see Table 1. Comparison with sands shows greater difference. For example, a friction angle of $32^{\circ}$ is identified from drained triaxial tests in the low pressure range $(P=100$ and $300 \mathrm{kPa})$ for loose Hostun sand [14], whereas [2] evaluate the friction angle of loose Karlsruhe sand at $34^{\circ}$. The mineral nature of each aggregate, the particle shape and surface roughness contribute to explain these differences.

\section{Conclusion}

This experimental study has helped elucidate the behaviour of loose, dry and moist sugar aggregates sheared at low confining pressures (below $100 \mathrm{kPa}$ ) in drained conditions. Test reproducibility is good in terms of stress-strain curves, contrarily to volumetric strain which is hard to reproduce at such low densities and pressures. No crushing of grains is noticed, which explains the independence of several intrinsic sugar parameters to confining pressure. Parameters are also insensitive to limited variations in low initial sample density (coefficient of variation on the order of $6 \%$ ). The initial normalised Young's modulus was shown to be pressure, strain rate and particle size dependent, but it does not noticeably vary with $10 \%$ moisture (sugar saturated water or glycerol). Concerning critical state, it 
is hard to confidently characterise from stress-strain curves and $\left(\varepsilon_{a}, \varepsilon_{v}\right)$ curves only. We propose to use Rowe's interpretation [5] in the dilatancy-rate/stress ratio plane and we show that critical state is effectively reached at an axial strain $\varepsilon_{a c v}$ on the order of $20 \%$ but varying from one test to another. Several parameters intrinsic to sugar $\left(K_{\mu}, K_{c v}\right)$ are identified and a statistically significant difference between their averages is characterised only between dry sugar and sugar moistened by $10 \%$ sugar saturated water at high strain rate. Corresponding angles of intergranular friction $\Phi_{\mu}$ and critical state friction angle $\varphi_{c v}$ are inferred. $\varphi_{c v}$ is $36^{\circ}$ at low strain rate, which matches Kolymbas \& al. results [2] in approaching test conditions. A particular feature of the shearing of relatively loose sugar seems to be that the blocky nature of the grains creates substantial dilatancy even though the stress ratio never exceeds the critical state value. A shear flow of loose sugar mobilising a critical state angle of internal friction seems to be able to exhibit a very wide range of

dilatancy-rates, $\left(\frac{d \varepsilon_{v}}{d \varepsilon_{a}}\right)$ between -1 and 0 . This may well give rise to instabilities in flow regimes.

\section{Acknowledgments}

The authors are grateful to the EPSRC (project n. GR/M76966) and to the industrial partners Thomas Broadbent \& Sons Ltd, British Sugar Plc and Tate \& Lyle Plc. 


\section{References}

[1] P. D. Thompson and G. C. Grimwood. Developments with high grade continuous centrifugal machines. In Technical Session 4C(i), EuroTechLink 97, England, 1997.

[2] D. Kolymbas and W. Wu. Recent Results of Triaxial Tests with Granular Materials. Powder Technology, 60:99-119, 1990.

[3] A. W. Bishop and D. J. Henkel. The Measurement of Soil Properties in the Triaxial Test. 2nd Ed., Edward Arnold, London, 1962.

[4] S. Kamath, V.M. Puri, H.B. Manbeck, and R. Hogg. Flow Properties of Powders using Four Testers - Measurement, Comparison and Assessment. Powder Technology, $76: 277-289,1993$.

[5] P. W. Rowe. Theoretical meaning and observed values of deformation parameters for soil. In Stress-strain behaviour of soils, Proceedings of the Roscoe Memorial Symposium, Cambridge University, Edited by H.G. Parry, 29-31 March, pages 143-194, 1971.

[6] M. F. Ashby and G. M. Pharr. On Creep Enhanced by a Liquid Phase. Acta Metallica, 23:129-138, 1983.

[7] ASTM International. D4254-00 Standard Test Methods for Minimum Index Density and Unit Weight of Soils and Calculation of Relative Density. U.S. book of standards, 2003.

[8] Z. Bubnik, P. Kadlec, D. Urban, and M. Bruhns. Sugar Technologists ManualChemical and Physical Data for Sugar Manufacturers and Users. Verlag Dr. Albert Bartens, Berlin, Germany, 1995. 
[9] E. Flavigny, J. Desrues, and B. Palayer. Note Technique - Le Sable de Hostun RF. Revue Française de Géotechnique, 53(octobre):67-70, 1990.

[10] J. Bertozzi. Comportement mécanique d'un sable homométrique stabilisé. Revue Française de Géotechnique, 60(juillet):51-59, 1992.

[11] M. D. Bolton. The strength and dilatancy of sands. Géotechnique, 36(1):65-78, 1986.

[12] J. H. Atkinson. An Introduction to the Mechanics of Soils and Foundations. Mc Graw-Hill International Series in Civil Engineering, London, 1993.

[13] D. J. White. PSD measurement using the single particle optical sizing (SPOS) method. Géotechnique, 53(3):317-326, 2003.

[14] E. Flavigny, P. Levebvre, and H. Bousquet. Répétabilité et reproductibilité des essais triaxiaux de révolution. In G.R.E.C.O. Rhéologie des Géomatériaux - Sols, bétons et roches, rapport scientifique, 1988.

[15] C. G. Paradine and B. H. P. Rivett. Statistical Methods for Technologists - An Introduction for Students, Research Workers and Engineers to the principles of Statistical Method and Theory. D. G. Christopherson, Gen. Ed., English Universities Press Ltd., London, 3rd Ed., 1964.

[16] M. D. Bolton. A Guide to Soil Mechanics. MacMillan Education Ltd, 1979.

[17] Y. P. Cheng, Y. Nakata, and M. D. Bolton. Discrete Element Simulation of crushable soil. Géotechnique, accepted, 2003.

[18] M. Abramowitz and I. A. Stegun. Handbook of Mathematical Functions with Formulas, Graphs and Mathematical Table. 10th Ed., Dover Pubns., 1974. 


\section{List of Figures}

1 (a): Triaxial loading of a specimen by a pressure $P$ and a superimposed uniaxial compressive stress $\sigma_{a}$. (b): The triaxial test arrangement used for the identification of the shearing behaviour of sugar crystals: a reflective laser device allows for the measurement of a length between two reflective targets, in the central zone of the specimen; an emission (E) and reception (R) laser device measures the diameter of the specimen in its central zone.

2 (a): SEM photograph of as-received extra-fine sugar crystals, manufactured by British Sugar Plc. (b): The grain size distributions of British Sugar and Tate \& Lyle sugar. (c): British Sugar sugar crystals after compressive triaxial testing such that $P=80 \mathrm{kPa}$ and $\dot{\varepsilon_{a}}=3.3 \% / \mathrm{sec}$. (d): Grain size distribution for British Sugar sugar before and after triaxial testing at $P=80 \mathrm{kPa}$ and $\dot{\varepsilon_{a}}=3.3 \% / \mathrm{sec} \ldots \ldots \ldots \ldots$

3 Reproducibility of the tests and effect of initial density upon triaxial tests: example of dry sugar at $\dot{\varepsilon_{a}}=0.16 \% /$ sec. . . . . . . . . . . . . . .

4 Triaxial tests for dry extra-fine sugar at axial strain rates of (a): $0.16 \% / \mathrm{sec}$ and $(\mathrm{b}): 3.3 \% / \mathrm{sec} \ldots \ldots \ldots \ldots$

5 Triaxial tests for extra-fine sugar mixed with either (a): 10\% glycerol and (b): $10 \%$ sugar saturated water, at $\dot{\varepsilon_{a}}=3.3 \% /$ sec. . . . . . . . . . . .

6 Determination of the initial normalized Young's modulus $E_{0} / \sigma_{3}$ : (a): two examples at $\dot{\varepsilon_{a}}=0.16 \% / \mathrm{sec}$. (b): Strain rate effect upon $E_{0} / \sigma_{3}$ versus $\sigma_{3}=P$ for dry sugar. (c): Particle size effect upon $E_{0} / \sigma_{3}$ versus $P$ at $\dot{\varepsilon_{a}}=3.3 \% / \mathrm{sec}$ (dry aggregates). (d): Moisture influence upon $E_{0} / \sigma_{3}$ versus $P$ for sugar $+10 \%$ glycerol and sugar $+10 \%$ sugar saturated water tested at $\dot{\varepsilon_{a}}=3.3 \% / \mathrm{sec}$

Rowe's interpretation of triaxial tests. (a): Sketch giving principal stress ratio $K$ versus rate of dilatancy $\left(\frac{d \varepsilon_{v}}{d \varepsilon_{a}}\right)$. Zone $(\alpha)$ corresponds to local grain collapse; Zone $(\beta)$ : coherent rearrangement (linear behaviour) and zone $(\gamma)$ : critical state (plateau). (b): Filtering of strain data is aimed at reducing noise on evaluating $\left(\frac{d \varepsilon_{v}}{d \varepsilon_{a}}\right)$. Example of dry sugar at $\dot{\varepsilon_{a}}=0.16 \% / \mathrm{sec}, P=$ $22 \mathrm{kPa}$ and $\gamma_{0}=9.68 \mathrm{kN} / \mathrm{m}^{3}$ : true axial strain versus time and true volumetric strain versus time. (c): Experimental data for $K$ versus $\left(\frac{d \varepsilon_{v}}{d \varepsilon_{a}}\right)$, example of dry sugar at $\dot{\varepsilon_{a}}=0.16 \% / \mathrm{sec}, P=22 \mathrm{kPa}$ and $\gamma_{0}=9.68 \mathrm{kN} / \mathrm{m}^{3} .31$ Identification method for $K_{c v}, \varepsilon_{a c v}$ (and also $K_{\mu}$ ) using $K$ and $\left(\frac{d \varepsilon_{v}}{d \varepsilon_{a}}\right)$ versus $\varepsilon_{a}$ : example on dry sugar at $\dot{\varepsilon_{a}}=0.16 \% / \mathrm{sec}, P=56 \mathrm{kPa}$ and $\gamma_{0}=$ $8.35 \mathrm{kN} / \mathrm{m}^{3} \ldots \ldots \ldots \ldots \ldots \ldots \ldots \ldots$ 
9 Comparison of parameters $K_{c v}$ and $K_{\mu}$; solid errorbars represent mean value of parameter $+/$ - one standard deviation; dashed errorbars represent mean value of parameter +/- two standard deviations. (a): Strain rate effect upon dry sugar behaviour. (b): Grain size effect upon dry sugar behaviour at $\dot{\varepsilon_{a}}=3.3 \% / \mathrm{sec}$. (c): Moisture effect (10\% glycerol) upon sugar behaviour at $\dot{\varepsilon_{a}}=3.3 \% / \mathrm{sec}$. (d): Moisture effect (10\% sugar saturated water) upon sugar behaviour at $\dot{\varepsilon_{a}}=3.3 \% /$ sec. . . . . . . . . . . . . . . 

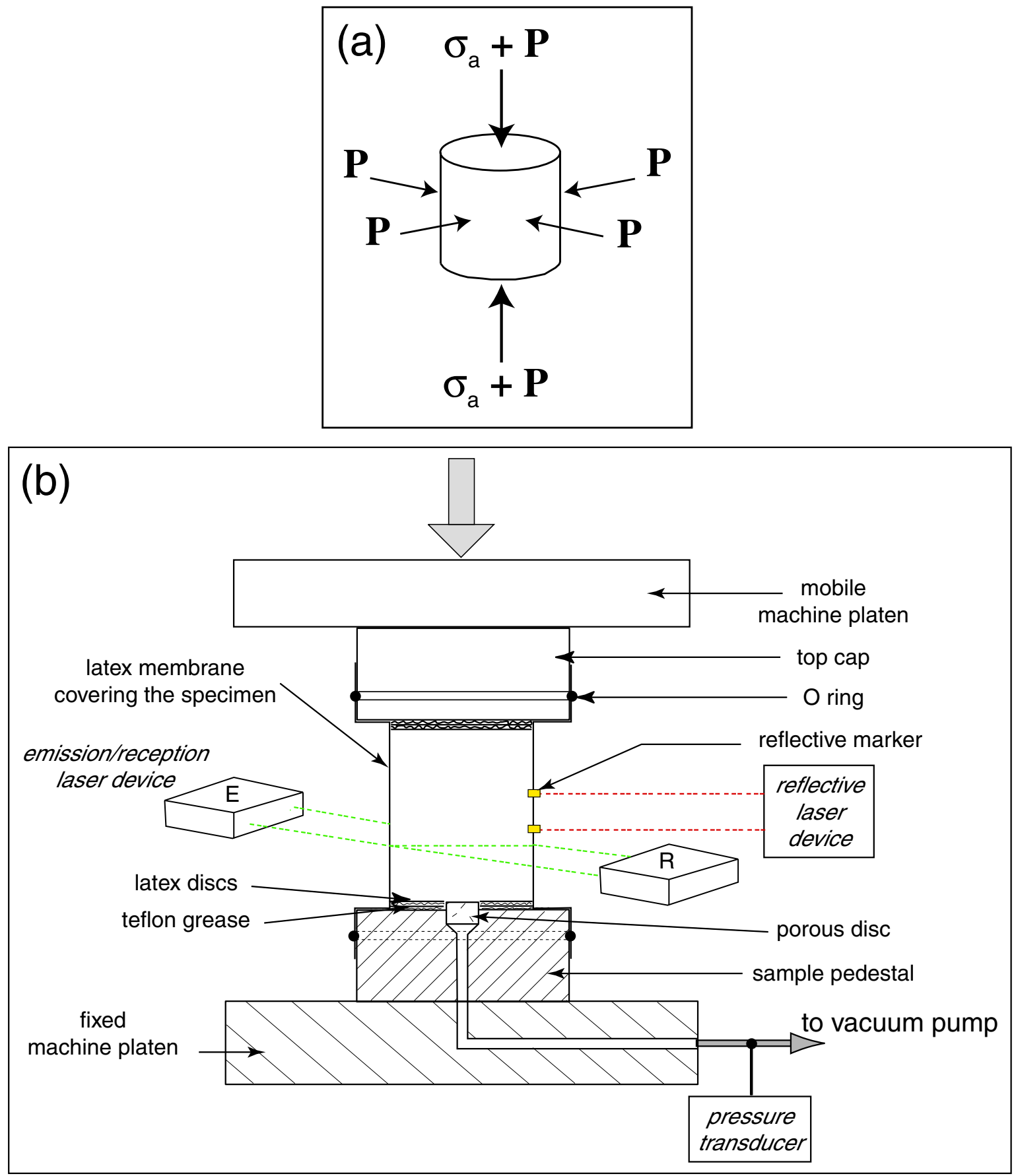

Figure 1: (a): Triaxial loading of a specimen by a pressure $P$ and a superimposed uniaxial compressive stress $\sigma_{a}$. (b): The triaxial test arrangement used for the identification of the shearing behaviour of sugar crystals: a reflective laser device allows for the measurement of a length between two reflective targets, in the central zone of the specimen; an emission $(\mathrm{E})$ and reception $(\mathrm{R})$ laser device measures the diameter of the specimen in its central zone. 


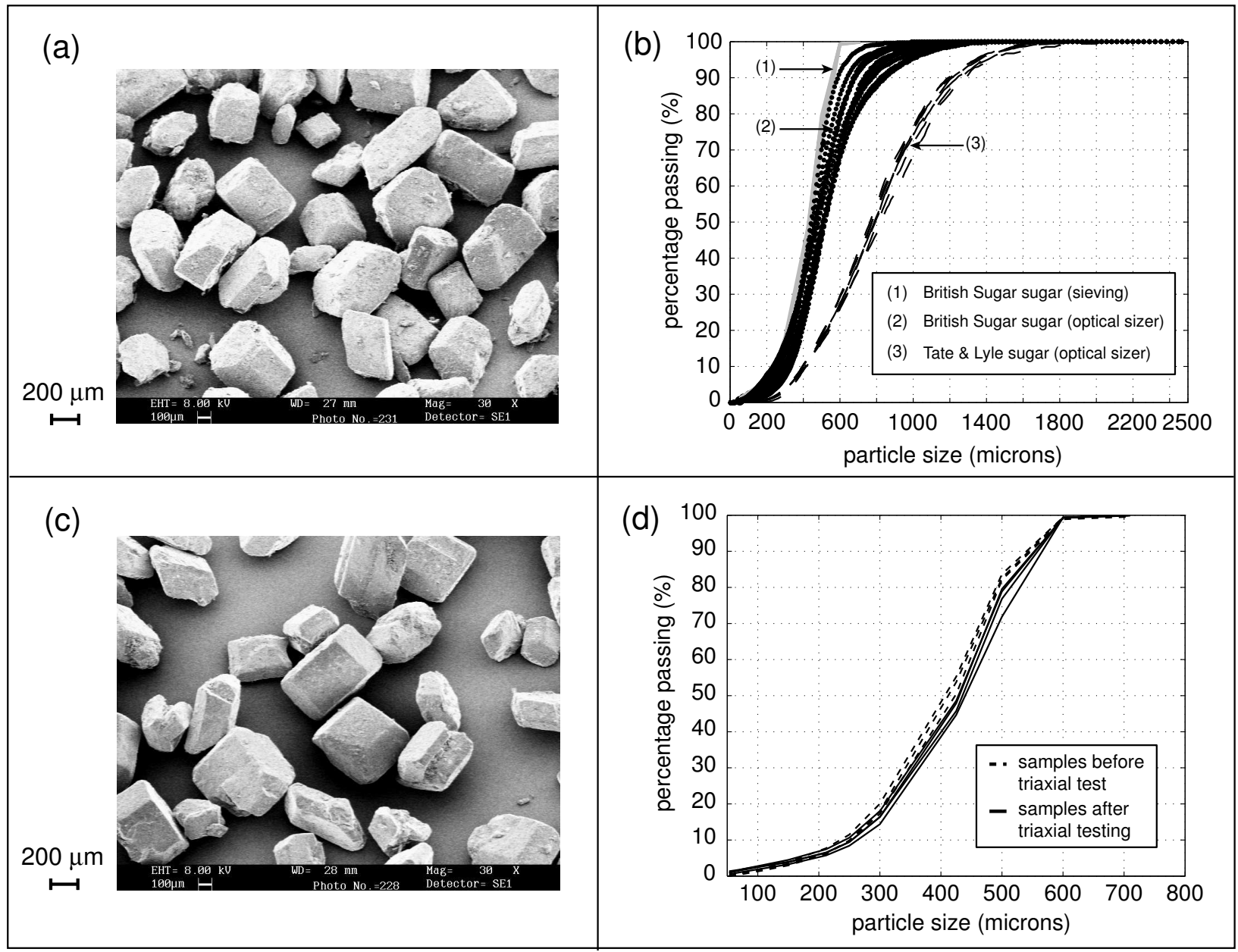

Figure 2: (a): SEM photograph of as-received extra-fine sugar crystals, manufactured by British Sugar Plc. (b): The grain size distributions of British Sugar and Tate \& Lyle sugar. (c): British Sugar sugar crystals after compressive triaxial testing such that $P=80 \mathrm{kPa}$ and $\dot{\varepsilon_{a}}=3.3 \% / \mathrm{sec}$. (d): Grain size distribution for British Sugar sugar before and after triaxial testing at $P=80 \mathrm{kPa}$ and $\dot{\varepsilon_{a}}=3.3 \% / \mathrm{sec}$. 

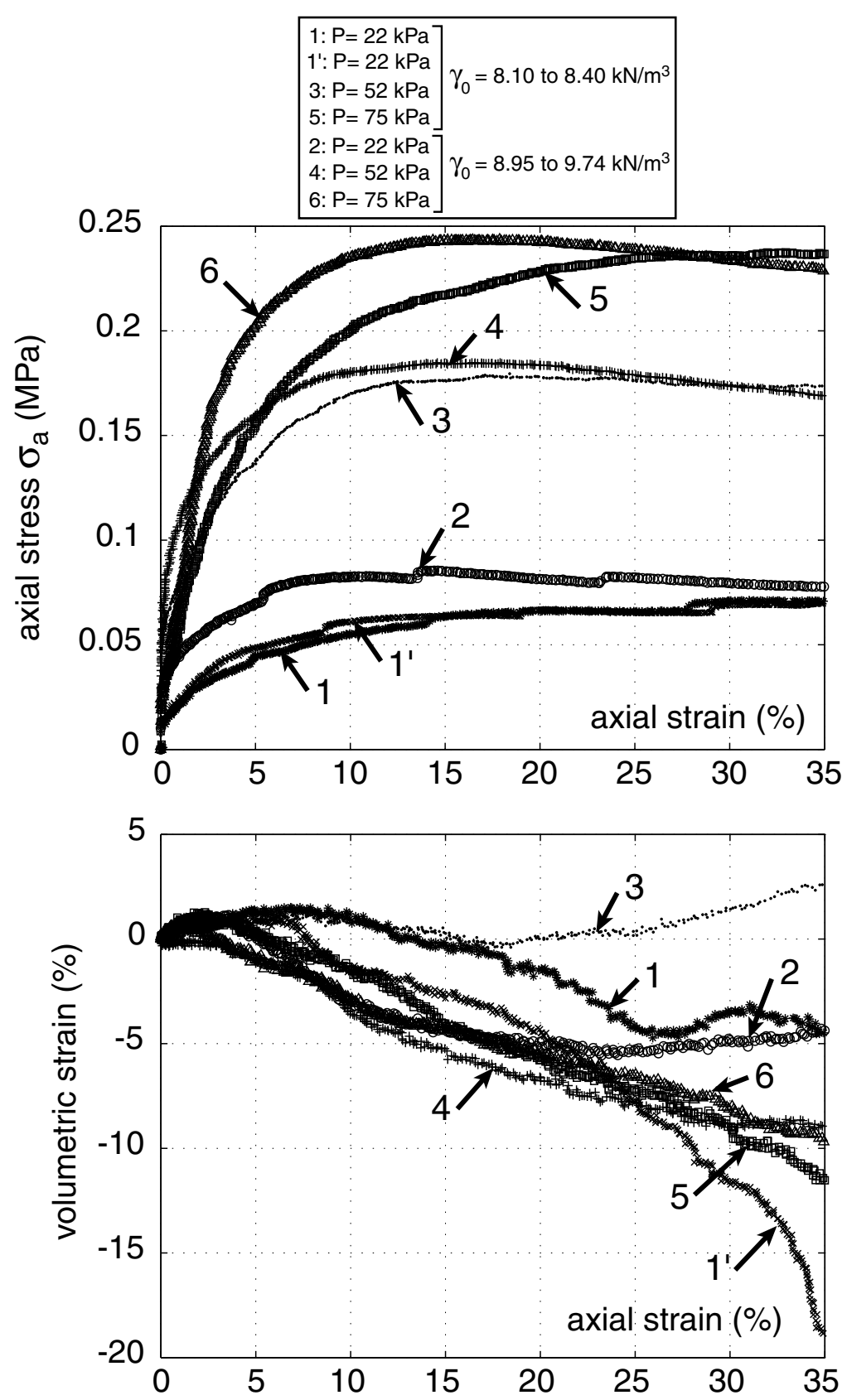

Figure 3: Reproducibility of the tests and effect of initial density upon triaxial tests: example of dry sugar at $\dot{\varepsilon_{a}}=0.16 \% /$ sec. 


$$
\text { (a) } \begin{gathered}
\dot{\varepsilon}_{\mathrm{a}}=0.16 \% / \mathrm{sec} \\
\text { 1: } \sigma_{3}=19 \mathrm{kPa} ; \gamma_{0}=8.10 \mathrm{kN} / \mathrm{m}^{3} \\
\text { 2: } \sigma_{3}=22 \mathrm{kPa} ; \gamma_{0}=8.11 \mathrm{kN} / \mathrm{m}^{3} \\
3: \sigma_{3}=56 \mathrm{kPa} ; \gamma_{0}=8.35 \mathrm{kN} / \mathrm{m}^{3} \\
4: \sigma_{3}=71 \mathrm{kPa} ; \gamma_{0}=8.74 \mathrm{kN} / \mathrm{m}^{3} \\
\text { 5: } \sigma_{3}=75 \mathrm{kPa} ; \gamma_{0}=8.07 \mathrm{kN} / \mathrm{m}^{3} \\
\text { 6: } \sigma_{3}=75 \mathrm{kPa} ; \gamma_{0}=8.95 \mathrm{kN} / \mathrm{m}^{3}
\end{gathered}
$$
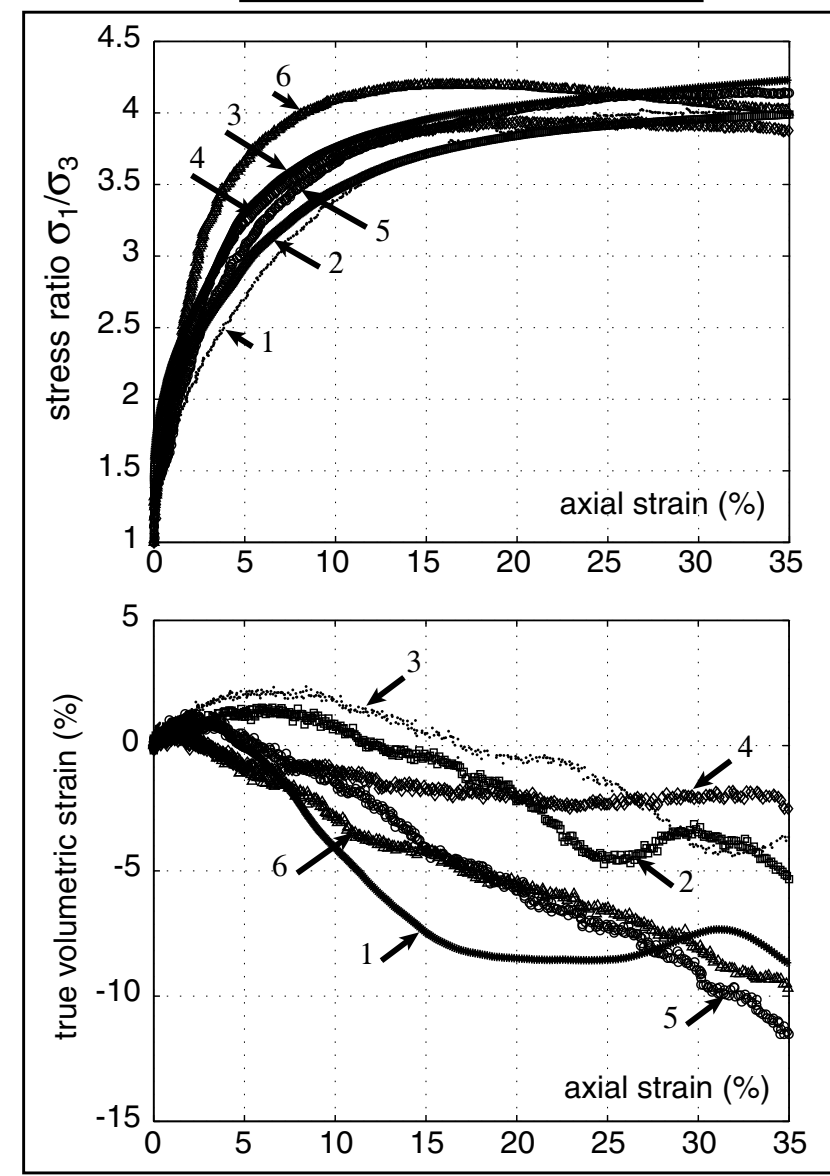

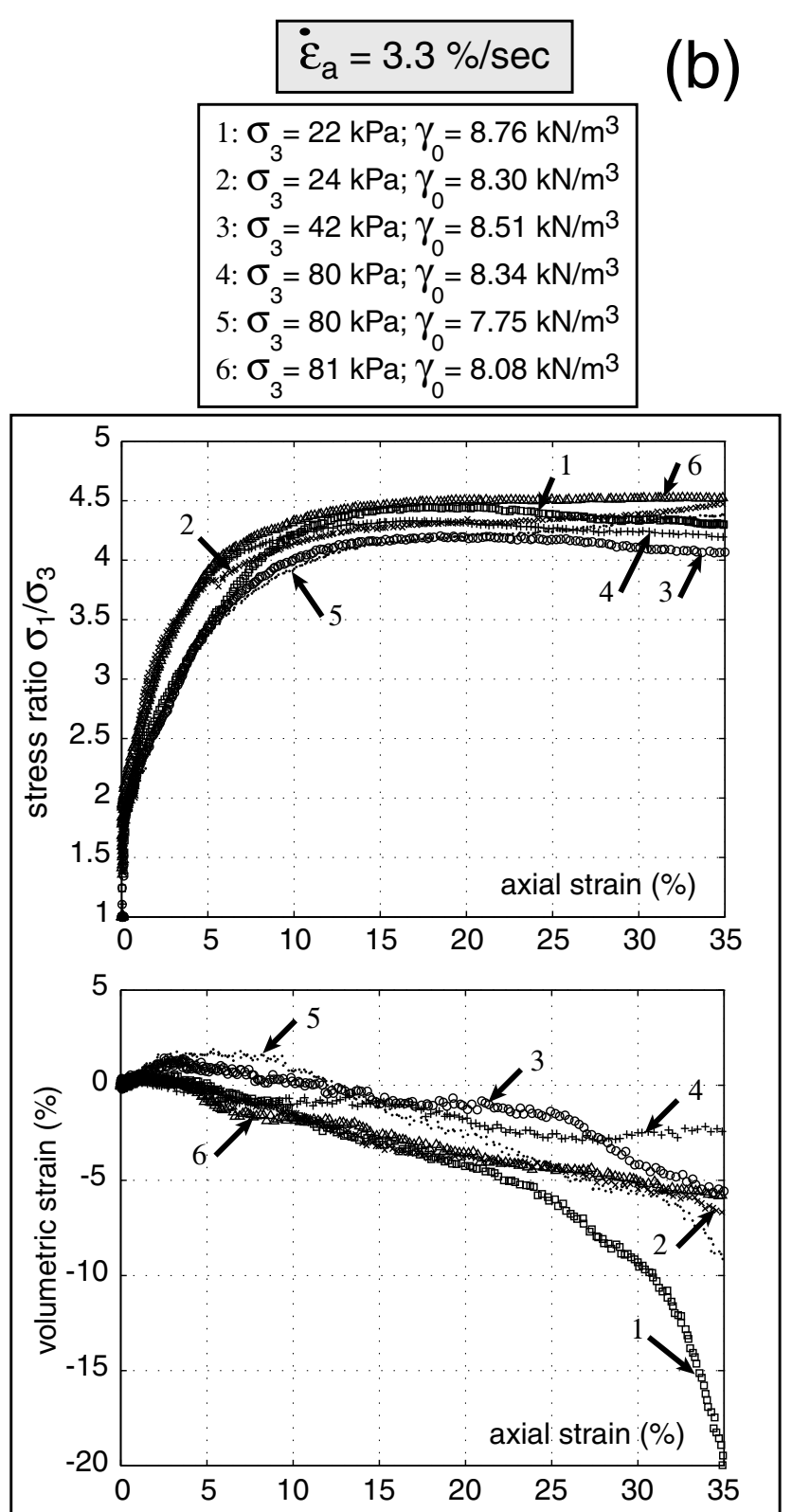

Figure 4: Triaxial tests for dry extra-fine sugar at axial strain rates of (a): $0.16 \% /$ sec and (b): $3.3 \% / \mathrm{sec}$. 


\section{(a)

\begin{tabular}{c} 
sugar \\
$+10 \%$ glycerol \\
\hline
\end{tabular}

$1: \sigma_{3}=23 \mathrm{kPa} ; \gamma_{0}=8.32 \mathrm{kN} / \mathrm{m}^{3}$

2: $\sigma_{3}=39 \mathrm{kPa} ; \gamma_{0}=8.58 \mathrm{kN} / \mathrm{m}^{3}$

3: $\sigma_{3}=59 \mathrm{kPa} ; \gamma_{0}=8.56 \mathrm{kN} / \mathrm{m}^{3}$

4: $\sigma_{3}=61 \mathrm{kPa} ; \gamma_{0}=8.53 \mathrm{kN} / \mathrm{m}^{3}$

$5: \sigma_{3}=79 \mathrm{kPa} ; \gamma_{0}=8.68 \mathrm{kN} / \mathrm{m}^{3}$

6: $\sigma_{3}=81 \mathrm{kPa} ; \gamma_{0}=7.29 \mathrm{kN} / \mathrm{m}^{3}$ sugar $+10 \%$ sugar saturated water

(b)

\section{$\dot{\varepsilon}_{\mathrm{a}}=3.3 \% / \mathrm{sec}$}

1: $\sigma_{3}=42 \mathrm{kPa} ; \gamma_{0}=7.77 \mathrm{kN} / \mathrm{m}^{3}$

2: $\sigma_{3}=79 \mathrm{kPa} ; \gamma_{0}=7.69 \mathrm{kN} / \mathrm{m}^{3}$

3: $\sigma_{3}=79 \mathrm{kPa} ; \gamma_{0}=8.83 \mathrm{kN} / \mathrm{m}^{3}$

4: $\sigma_{3}=80 \mathrm{kPa} ; \gamma_{0}=7.53 \mathrm{kN} / \mathrm{m}^{3}$

$5: \sigma_{3}=82 \mathrm{kPa} ; \gamma_{0}=7.90 \mathrm{kN} / \mathrm{m}^{3}$
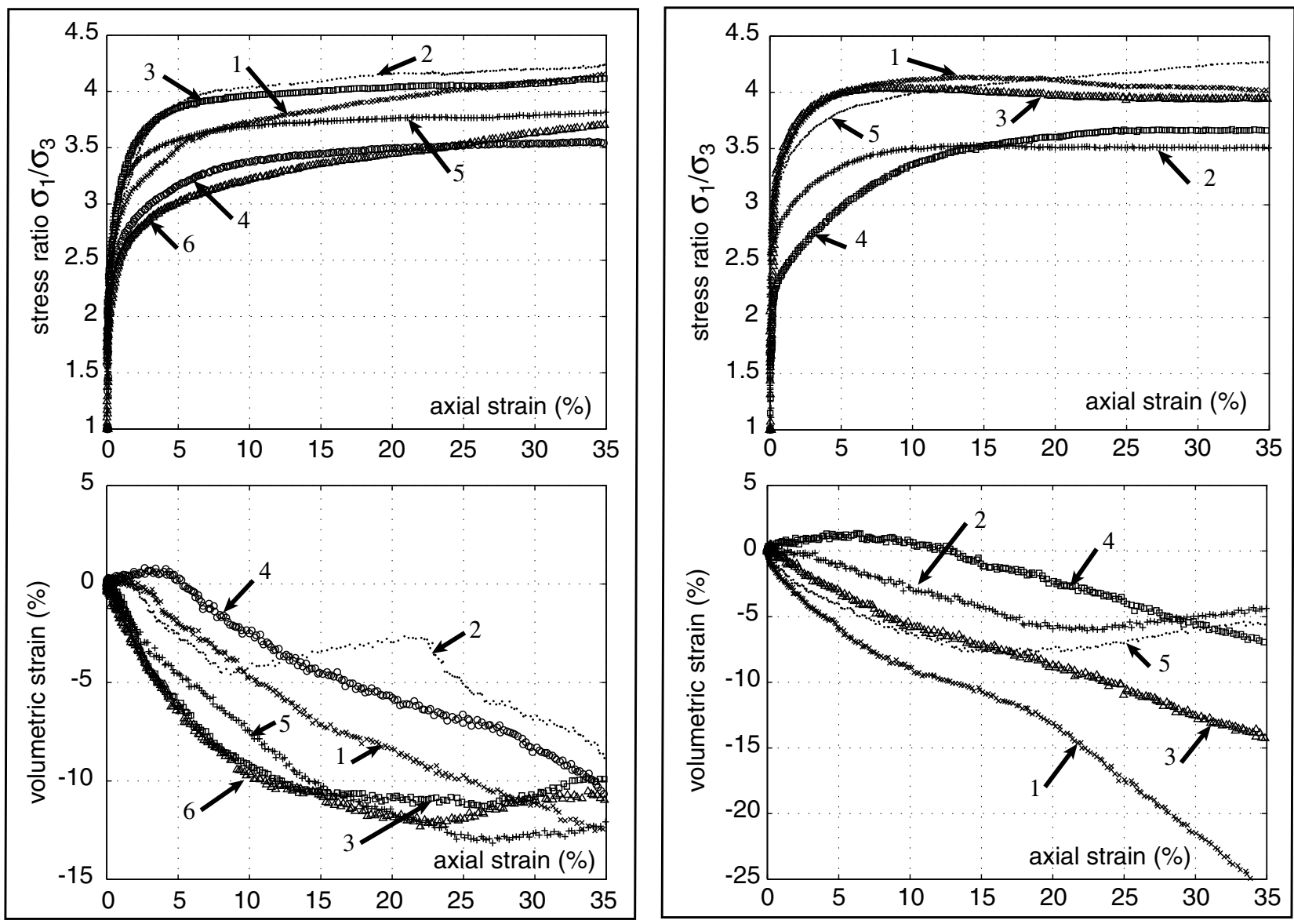

Figure 5: Triaxial tests for extra-fine sugar mixed with either (a): 10\% glycerol and (b): 10\% sugar saturated water, at $\dot{\varepsilon_{a}}=3.3 \% / \mathrm{sec}$. 


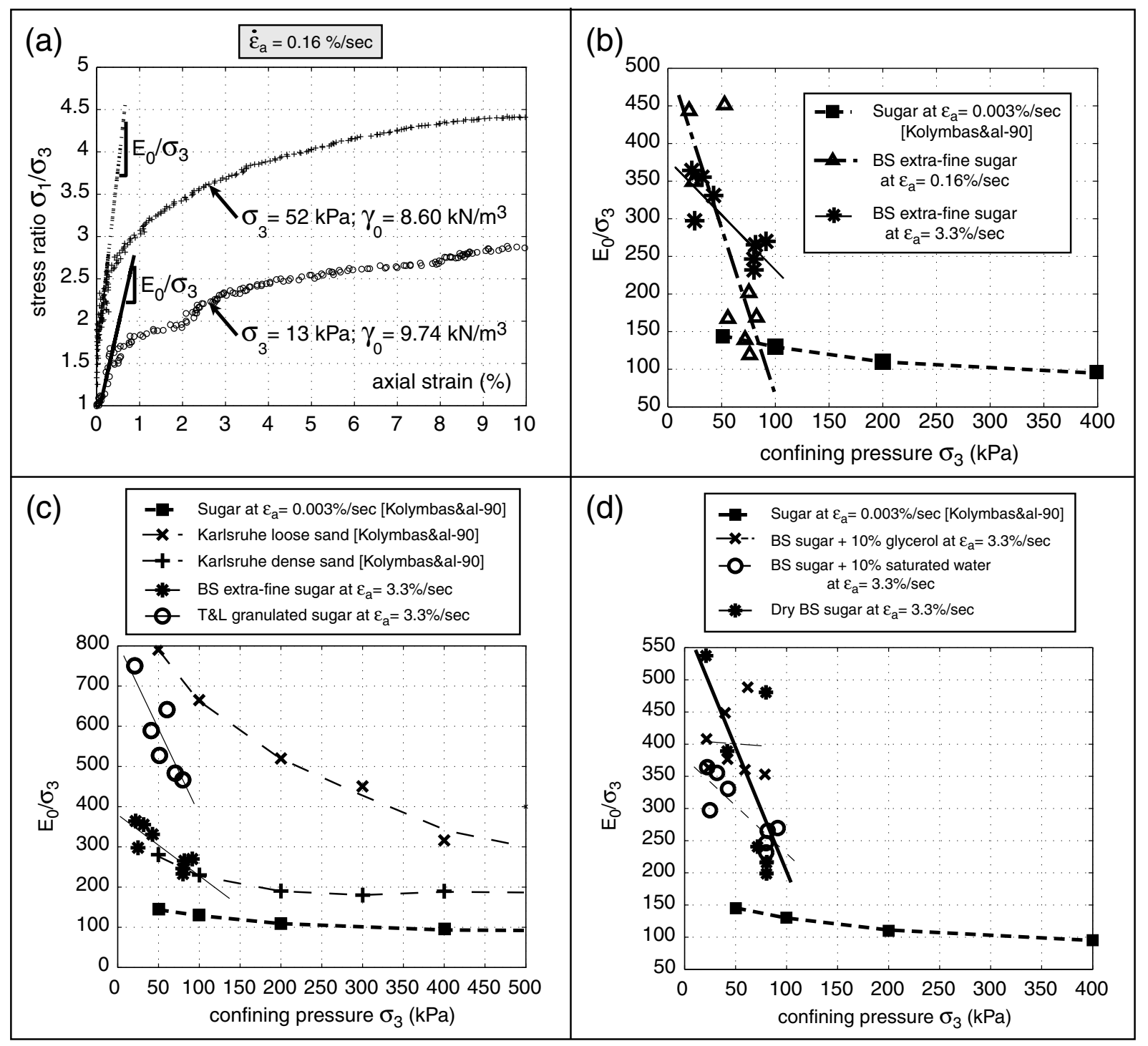

Figure 6: Determination of the initial normalized Young's modulus $E_{0} / \sigma_{3}$ : (a): two examples at $\dot{\varepsilon_{a}}=0.16 \% / \mathrm{sec}$. (b): Strain rate effect upon $E_{0} / \sigma_{3}$ versus $\sigma_{3}=P$ for dry sugar. (c): Particle size effect upon $E_{0} / \sigma_{3}$ versus $P$ at $\dot{\varepsilon_{a}}=3.3 \% /$ sec (dry aggregates). $(d)$ : Moisture influence upon $E_{0} / \sigma_{3}$ versus $P$ for sugar $+10 \%$ glycerol and sugar $+10 \%$ sugar saturated water tested at $\dot{\varepsilon_{a}}=3.3 \% / \mathrm{sec}$. 

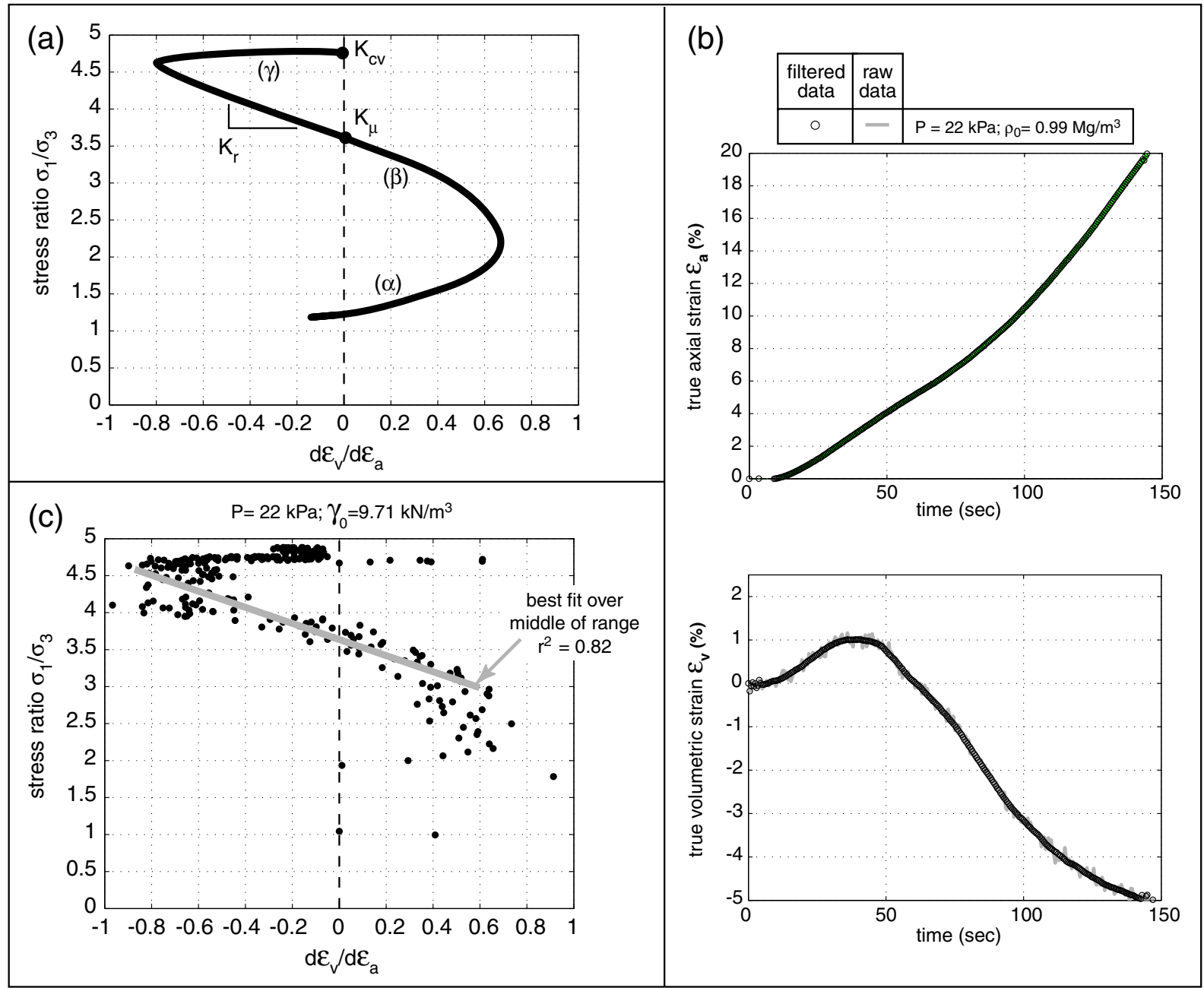

Figure 7: Rowe's interpretation of triaxial tests. (a): Sketch giving principal stress ratio $K$ versus rate of dilatancy $\left(\frac{d \varepsilon_{v}}{d \varepsilon_{a}}\right)$. Zone $(\alpha)$ corresponds to local grain collapse; Zone $(\beta)$ : coherent rearrangement (linear behaviour) and zone $(\gamma)$ : critical state (plateau). (b): Filtering of strain data is aimed at reducing noise on evaluating $\left(\frac{d \varepsilon_{v}}{d \varepsilon_{a}}\right)$. Example of dry sugar at $\dot{\varepsilon_{a}}=0.16 \% / \mathrm{sec}, P=22 \mathrm{kPa}$ and $\gamma_{0}=9.68 \mathrm{kN} / \mathrm{m}^{3}$ : true axial strain versus time and true volumetric strain versus time. (c): Experimental data for $K$ versus $\left(\frac{d \varepsilon_{v}}{d \varepsilon_{a}}\right)$, example of dry sugar at $\dot{\varepsilon_{a}}=0.16 \% / \mathrm{sec}, P=22 \mathrm{kPa}$ and $\gamma_{0}=9.68 \mathrm{kN} / \mathrm{m}^{3}$. 


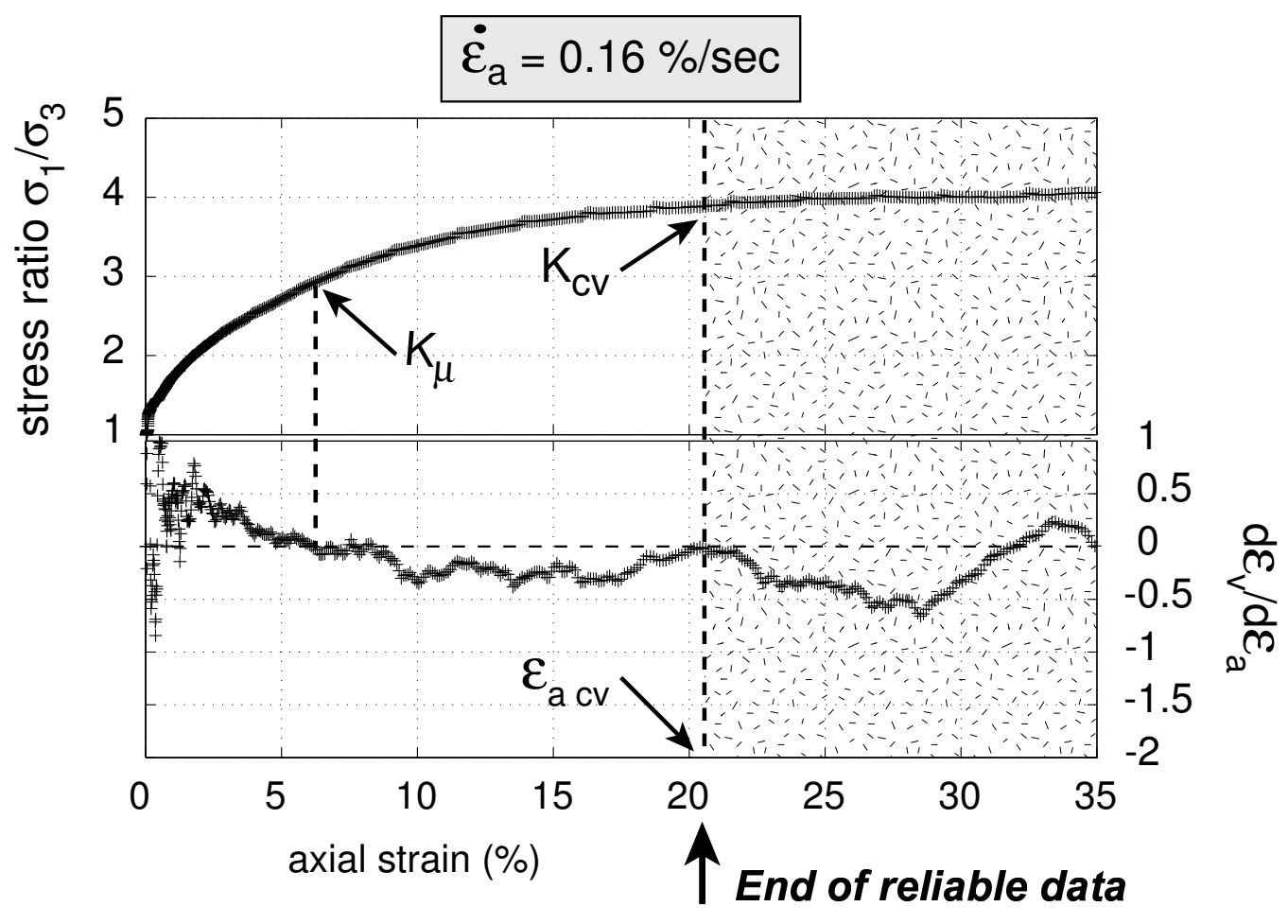

Figure 8: Identification method for $K_{c v}, \varepsilon_{a c v}$ (and also $K_{\mu}$ ) using $K$ and $\left(\frac{d \varepsilon_{v}}{d \varepsilon_{a}}\right)$ versus $\varepsilon_{a}$ : example on dry sugar at $\dot{\varepsilon_{a}}=0.16 \% / \mathrm{sec}, P=56 \mathrm{kPa}$ and $\gamma_{0}=8.35 \mathrm{kN} / \mathrm{m}^{3}$. 


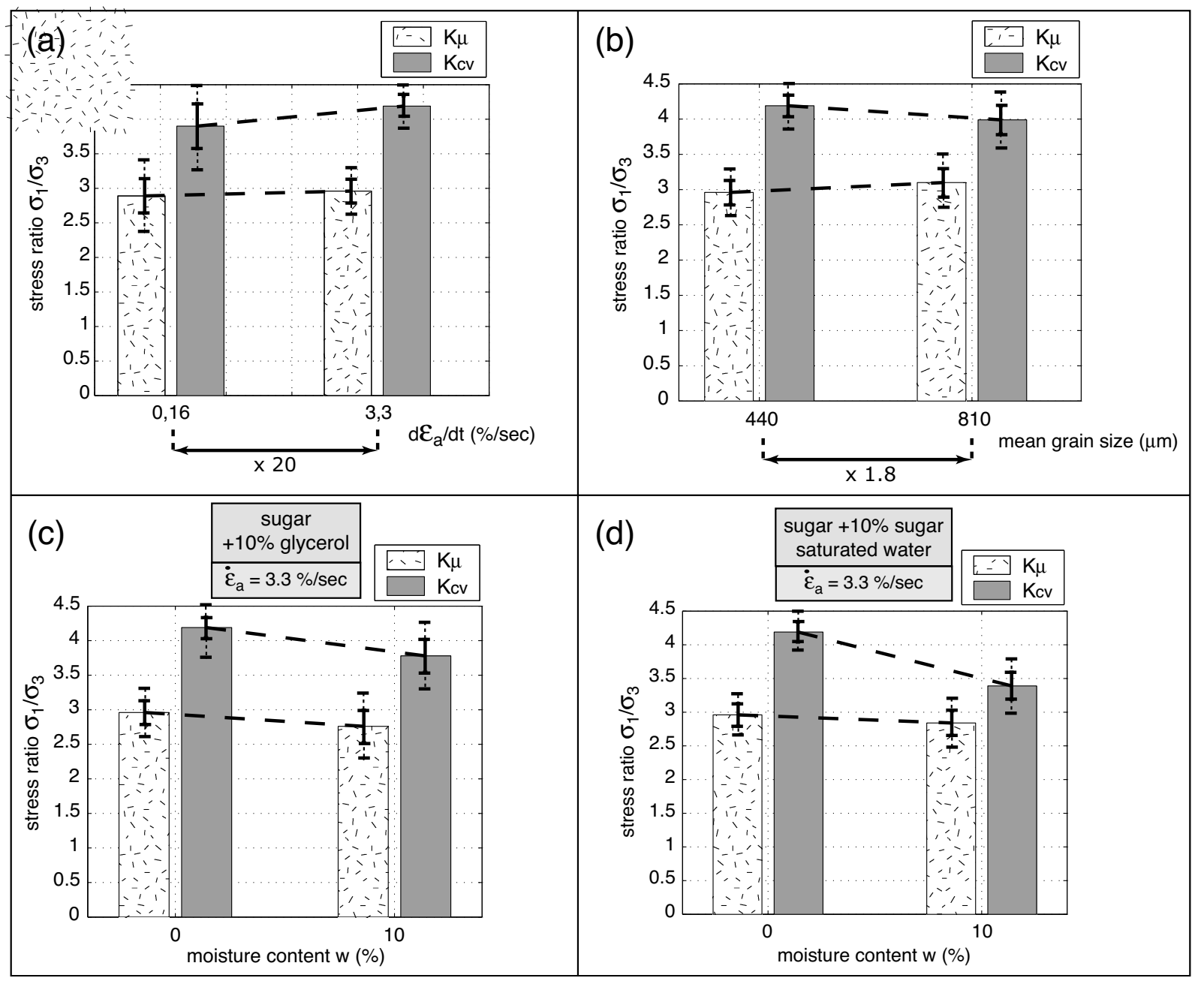

Figure 9: Comparison of parameters $K_{c v}$ and $K_{\mu}$; solid errorbars represent mean value of parameter +/- one standard deviation; dashed errorbars represent mean value of parameter +/- two standard deviations. (a): Strain rate effect upon dry sugar behaviour. (b): Grain size effect upon dry sugar behaviour at $\dot{\varepsilon_{a}}=3.3 \% / \mathrm{sec}$. (c): Moisture effect (10\% glycerol) upon sugar behaviour at $\dot{\varepsilon_{a}}=3.3 \% / \mathrm{sec}$. (d): Moisture effect (10\% sugar saturated water) upon sugar behaviour at $\dot{\varepsilon_{a}}=3.3 \% / \mathrm{sec}$. 


\section{List of Tables}

1 Main features of the particle characteristics (BS stands for British Sugar and TL for Tate \& Lyle) test procedure and interpretation, and comparison with $[2]$ and $[4] \ldots \ldots \ldots \ldots \ldots$

2 Results on dry sugars for initial normalized Young's modulus and linear

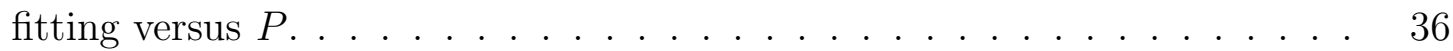

3 Results for $K_{\mu}, \varphi_{\mu}, K_{c v}$ and $\varphi_{c v}$ for the 5 types of tests performed. . . . . 37 


\begin{tabular}{|c|c|c|c|}
\hline authors & this paper & Kolymbas \& al & Kamath \& al \\
\hline $\begin{array}{l}\gamma_{0} \text { range } \\
\left(\mathrm{kN} / \mathrm{m}^{3}\right)\end{array}$ & $7.25-9.74$ & 8.46-9.49 & 9.04 \\
\hline$e_{0 \min } ; e_{0 \max }$ & $0.60-1.15$ & $0.64-0.84$ & not given \\
\hline $\begin{array}{l}\text { mean grain size } \\
\qquad(\mu \mathrm{m})\end{array}$ & $\begin{array}{l}440(\mathrm{BS}) \\
810(\mathrm{TL})\end{array}$ & 430 & 542.8 \\
\hline $\begin{array}{c}\text { sample dimensions } \\
\qquad H_{0} ; D_{0}\end{array}$ & $5 \mathrm{~cm} ; 5 \mathrm{~cm}$ & $10 \mathrm{~cm} ; 10 \mathrm{~cm}$ & $H_{0} / D_{0}=2.3$ \\
\hline $\begin{array}{c}\text { displacement rate } \\
\dot{d}(\mathrm{~mm} / \mathrm{min})\end{array}$ & $5 ; 100$ & $(1 / 6)$ & 1 \\
\hline$P$ range $(\mathrm{kPa})$ & $13-95$ & $\begin{array}{c}50 ; 100 ; \\
200 \text { and over }\end{array}$ & $24.1 ; 31.0 ; 37.9$ \\
\hline number of tests & 54 & at least 10 & 9 \\
\hline comments & $\begin{array}{l}\text { lubricated } \\
\text { ends }\end{array}$ & $\begin{array}{l}\text { lubricated } \\
\text { ends }\end{array}$ & $\begin{array}{c}\text { non lubricated } \\
\text { ends }\end{array}$ \\
\hline $\begin{array}{l}\text { limit state } \\
\text { criterion }\end{array}$ & $\begin{array}{c}\text { Rowe's } \\
\text { interpretation } \\
\text { (see paper) }\end{array}$ & $\begin{array}{l}\text { axial strain } \\
=10 \%\end{array}$ & $\begin{array}{c}\text { axial strain } \\
\quad=15 \% \\
\text { or }\left(\sigma_{1}-\sigma_{3}\right)_{\max }\end{array}$ \\
\hline
\end{tabular}

Table 1: Main features of the particle characteristics (BS stands for British Sugar and TL for Tate \& Lyle) test procedure and interpretation, and comparison with [2] and [4]. 


\begin{tabular}{|c|c|c|c|}
\hline Test type & $\begin{array}{c}\text { Linear } \\
\text { interpolation } \\
\left(E_{0} / \sigma_{3}\right)=a \sigma_{3}+b\end{array}$ & $\begin{array}{c}\text { Coefficient } \\
\text { of } \\
\text { correlation } \\
\mathrm{r}^{2}\end{array}$ & $\begin{array}{c}\text { Number } \\
\text { of } \\
\text { tests }\end{array}$ \\
\hline \hline dry BS sugar & $\mathrm{a}=-4.50$ & 0.62 & \\
at $\dot{\varepsilon_{a}}=0.16 \% / \mathrm{sec}$ & $\mathrm{b}=512$ & Acceptable & 8 \\
\hline dry BS sugar & $\mathrm{a}=-1.46$ & 0.72 & 8 \\
at $\dot{\dot{\varepsilon}_{a}}=3.3 \% / \mathrm{sec}$ & $\mathrm{b}=378$ & Good & 8 \\
\hline dry TL sugar & $\mathrm{a}=-4.34$ & 0.73 & \\
at $\dot{\varepsilon_{a}}=3.3 \% / \sec$ & $\mathrm{b}=811$ & Good & 6 \\
\hline
\end{tabular}

Table 2: Results on dry sugars for initial normalized Young's modulus and linear fitting versus $P$. 


\begin{tabular}{|c|c|c|c|c|c|c|}
\hline $\begin{array}{c}\text { Test } \\
\text { conditions }\end{array}$ & $K_{\mu}$ & $\begin{array}{l}\text { Coefficient } \\
\text { of } \\
\text { variation } \\
(\%)\end{array}$ & $\begin{array}{c}\text { Friction } \\
\text { angle } \\
\varphi_{\mu} \\
\text { (deg) }\end{array}$ & $K_{c v}$ & $\begin{array}{l}\text { Coefficient } \\
\text { of } \\
\text { variation } \\
(\%)\end{array}$ & $\begin{array}{c}\text { Friction } \\
\text { angle } \\
\varphi_{c v} \\
\text { (deg) }\end{array}$ \\
\hline $\begin{array}{c}\text { dry BS sugar } \\
\text { at } \dot{\varepsilon_{a}}=0.16 \% / \mathrm{sec}\end{array}$ & 2.89 & 8.6 & 29.1 & 3.90 & 8.4 & 36.3 \\
\hline $\begin{array}{c}\text { dry BS sugar } \\
\text { at } \dot{\varepsilon_{a}}=3.3 \% / \mathrm{sec}\end{array}$ & 2.96 & 5.7 & 29.7 & 4.19 & 3.5 & 37.9 \\
\hline $\begin{array}{c}\text { dry TL sugar } \\
\text { at } \dot{\varepsilon_{a}}=3.3 \% / \mathrm{sec}\end{array}$ & 3.10 & 6.1 & 30.8 & 3.99 & 4.9 & 36.8 \\
\hline $\begin{array}{c}\text { sugar }+10 \% \\
\text { glycerol } \\
\text { at } \dot{\varepsilon_{a}}=3.3 \% / \mathrm{sec}\end{array}$ & 2.76 & 8.5 & 27.8 & 3.78 & 6.2 & 35.5 \\
\hline $\begin{array}{c}\text { sugar }+10 \% \text { sugar } \\
\text { saturated water } \\
\text { at } \dot{\varepsilon_{a}}=3.3 \% / \mathrm{sec}\end{array}$ & 2.84 & 6.4 & 28.6 & 3.39 & 6.0 & 32.9 \\
\hline
\end{tabular}

Table 3: Results for $K_{\mu}, \varphi_{\mu}, K_{c v}$ and $\varphi_{c v}$ for the 5 types of tests performed. 